\title{
Left ventricular dysfunction: a clue to cognitive impairment in older patients with heart failure
}

Giuseppe Zuccalà, Caterina Cattel, Ester Manes-Gravina, Maria G Di Niro, Alberto Cocchi, Roberto Bernabei

\begin{abstract}
Objectives-Cognitive impairment has been reported in middle aged patients with end stage heart failure. This cross sectional study assessed the prevalence and determinants of cognitive dysfunction in older patients with mild to moderate heart failure.

Methods-57 consecutive patients (mean age 76.7 years) with chronic heart failure underwent physical examination, blood chemistry, urinalysis, chest radiography ECG, Doppler echocardiography, and the mini mental state examination (MMSE), mental deterioration battery, depression scale of the Center for Epidemiological Studies (CES-D), Katz activities of daily living, and instrumental activities of daily living 24 hours before hospital discharge. Results-MMSE scores < 24 were found in $53 \%$ of participants. The MMSE score was associated with left ventricular ejection fraction according to a non-linear correlation, so that cognitive performance was significantly lower in subjects with left ventricular ejection fraction $\leqslant 30 \%$. The same pattern of correlation was evidenced between left ventricular ejection fraction and both the attention sub-item of MMSE and the Raven test score. In a multivariate linear regression model, after adjusting for age, sex, and a series of clinical data and objective tests, both age $(\beta=-0.30$; $P=0.038)$ and the natural log of left ventricular ejection fraction $(\beta=0.58 ; P=0.001)$ were associated with the MMSE score.

Conclusion-Cognitive impairment in older patients with chronic heart failure is common, and independently associated with lower left ventricular ejection fraction. Given the overwhelming incidence and prevalence of heart failure in older populations, early detection of cognitive impairment in these subjects with prompt, intensive treatment of left ventricular systolic dysfunction may prevent or delay a remarkable proportion of dementia in advanced age.
\end{abstract}

(F Neurol Neurosurg Psychiatry 1997;63:509-512)
Keywords: cognitive impairment; heart failure; left ventricular function

During recent years, heart failure has become the most common cause of hospital admission among geriatric patients. ${ }^{12}$ Some studies reported an abnormal prevalence of cognitive dysfunction in middle aged patients with end stage heart failure. ${ }^{3-5}$ The issue of so called "cardiogenic dementia" ${ }^{7}$ has been renewed by recent findings on the effects of pacemaker implantation and cardiac transplantation on cognitive function; $;^{38}$ in some studies, increasing age $^{5}$ and lower indices of left ventricular function $^{3}$ were associated with worsening cognitive performance. However, the linkage between aging, left ventricular systolic function, and cognitive performance remains elusive $^{3-5}$ 8-10 $^{-10}$ The present study aimed to assess the impact of older age and left ventricular systolic dysfunction on cognitive performance of patients with mild to moderate heart failure.

\section{Materials and methods}

From 1 January to 30 June 1996, 92 patients with a history of heart failure lasting four months or more were admitted to our geriatric department. Subjects who met the NINCDS/ ADRDA criteria ${ }^{11}$ for Alzheimer's disease (10 patients), with an Hachinski ischaemic score ${ }^{12}$ $>4$ (nine patients), inadequate echocardiographic studies (six patients), or with a history or present evidence of psychiatric disorders, alcohol misuse, hyperthyroidism, or having psychoactive therapy (10 patients) were excluded. Thus the study population comprised 57 patients (36 with coronary artery disease, 21 with both hypertensive and ischaemic heart disease) in the II-III New York Heart Association classes; the table shows their main characteristics. All participants underwent detailed physical and neurological evaluation 24 hours before hospital discharge. Cognitive abilities were assessed by means of the mini mental state examination (MMSE) ${ }^{13}$ and mental deterioration battery (MDB). ${ }^{14}$ The last scale explores six cognitive domains: verbal intelligence, short term verbal memory, immediate visual-spatial memory, and visual-spatial and constructional ability; visual-spatial intelli- 
Main characteristics of enrolled patients $(n=57)$ and association with MMSE score in multiple linear regression initial models

\begin{tabular}{|c|c|c|c|}
\hline & Mean (SEM) or $n(\%)$ & $\beta^{\star}$ & Pvalue \\
\hline \multicolumn{4}{|l|}{ Demographic data: } \\
\hline Age (y) & $77(1)$ & -0.31 & 0.024 \\
\hline Sex (female) & $41(72 \%)$ & -0.01 & 0.934 \\
\hline \multicolumn{4}{|l|}{ Clinical data: } \\
\hline Comorbid conditions & $5.4(0.24)$ & -0.22 & 0.091 \\
\hline ADLs & $34(60 \%) \dagger$ & 0.15 & 0.265 \\
\hline IADLs & $44(77 \%) \dagger$ & 0.32 & 0.076 \\
\hline Heart failure score & $7.5(0.4)$ & 0.17 & 0.364 \\
\hline New York Heart Association class & $3(1)$ & -0.21 & 0.219 \\
\hline Heart failure duration (months) & $35(5)$ & 0.09 & 0.485 \\
\hline Education (years) & $8.3(0.6)$ & 0.09 & 0.587 \\
\hline Use of ACE inhibitors & $33(58 \%)$ & 0.01 & 0.949 \\
\hline \multicolumn{4}{|l|}{ Objective tests: } \\
\hline Body mass index & $25.3(0.8)$ & 0.33 & 0.171 \\
\hline Left ventricular ejection fraction (\%) & $44.7(2.3)$ & $0.62 \ddagger$ & 0.005 \\
\hline Haemoglobin $(\mathrm{g} / \mathrm{l})$ & $123(3)$ & 0.21 & 0.400 \\
\hline Serum K $(\mathrm{mmol} / \mathrm{l})$ & $4.1(0.08)$ & 0.11 & 0.665 \\
\hline Serum Na $(\mathrm{mmol} / \mathrm{l})$ & $138.6(0.55)$ & 0.28 & 0.247 \\
\hline Serum Ca $(\mathrm{mmol} / \mathrm{l})$ & $2.35(0.02)$ & -0.13 & 0.668 \\
\hline Creatinine $(\mu \mathrm{mol} / \mathrm{l})$ & $141(13)$ & 0.50 & 0.032 \\
\hline Cholesterol $(\mathrm{mmol} / \mathrm{l})$ & $4.73(0.15)$ & 0.07 & 0.724 \\
\hline Serum cortisol $(\mu \mathrm{g} / \mathrm{l})$ & $199(13)$ & 0.10 & 0.660 \\
\hline Albumin $(g / 1)$ & $43(0.5)$ & -0.43 & 0.207 \\
\hline Systolic blood pressure $(\mathrm{mm} \mathrm{Hg})$ & $144(12)$ & 0.33 & 0.326 \\
\hline CES-D score & $24(1.8)$ & -0.34 & 0.190 \\
\hline
\end{tabular}

* Multiple linear regression initial models.

†Patients with impairment in one or more activities.

$\ddagger$ After natural log transformation. fraction and MMSE score $(F=2.33 ; \mathrm{P}=0.032)$, but not for the correlation between the natural log of left ventricular ejection fraction and the MMSE score $(F=1.76 ; \mathrm{P}=0.105)$. To assess independent associations with MMSE score, separate sex and age adjusted linear regression models were calculated for the groups of variables (clinical data and objective measurements, table). Variances of all continuous variables were normal, as assessed by one sample Kolmogorov-Smirnov test. Linearity of the correlation between age and MMSE score was evidenced by the ANOVA tests of linearity $(F=5.45 ; \mathrm{P}=0.027)$ and deviation from linearity $(F=0.91 ; \mathrm{P}=0.588)$. Among pharmacological variables, only the use of ACE inhibitors was considered, as patients on $\beta$ blocking treatment and those who did not use digitalis or diuretic drugs were insufficient for analysis. Eventually, those variables, significant at the $\mathrm{P}<0.01$ level in the initial models, were entered into a multivariate sex and age corrected summary model.

\section{Results}

The mean MMSE score was 23 (0.8); scores below 24 were detected in 30 patients (53\%). According to correlation coefficient calculations, lower MMSE scores were associated with decreasing cholesterol and serum sodium concentrations $(r=0.30 ; \mathrm{P}=0.02$, and $r=0.13$, $\mathrm{P}=0.04$ respectively), increasing age $(r=-0.31$; $\mathrm{P}=0.02)$, higher New York Heart Association classification $(r=-0.51 ; \mathrm{P}=0.001)$, lower left ventricular ejection fraction $\quad(r=0.38$; $\mathrm{P}=0.007$ ), and with decreased ability in both ADLs $(r=0.57 ; \mathrm{P}=0.001)$ and IADLs $(r=0.43$; $\mathrm{P}=0.003)$. However, analysis of several regression coefficients disclosed a non-linear relation between left ventricular and MMSE score, with a distinct fall in MMSE scores in patients whose ejection fraction values were below 30\% (figure); the cubic equation best fitted this relation $\left(R^{2}=0.42 ; \mathrm{P}<0.001\right)$. The same pattern of correlation was found between left ventricular ejection fraction and both the attention subitem of MMSE $\left(R^{2}=0.26 ; \mathrm{P}=0.001\right)$, and the performance scores in the Raven test $\left(\mathrm{R}^{2}=0.28 ; \quad \mathrm{P}<0.01\right)$; no associations were

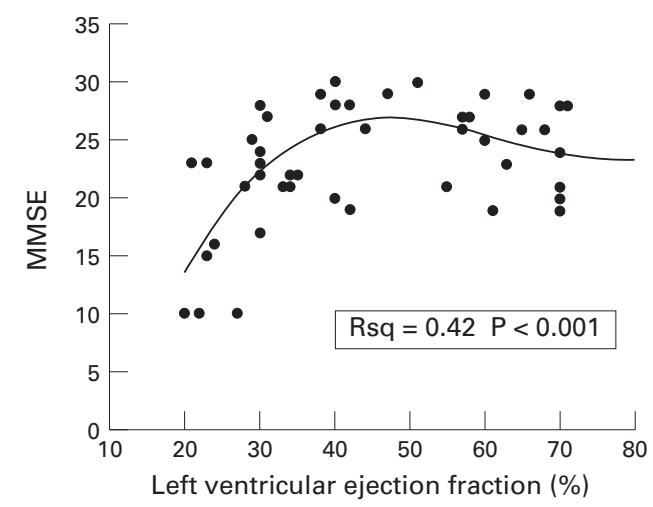

Distribution of MMSE scores according to left ventricular ejection fraction. The regression line describes a cubic equation, which best fitted the relation between these variables. Analysis of this regression model indicated a significant decrease in MMSE scores in patients with ejection fraction values $<30 \%$. 
detectable between left ventricular systolic function and the other items of MMSE, as well as with performance scores in other tasks of the MDB. Eventually, no association was found between left ventricular ejection fraction and age.

In initial regression models, increased heart failure severity score, decreased ability in IADLs, serum creatinine, and lower left ventricular ejection fraction (as natural logarithm) were associated with lower MMSE scores, after correction for covariates (table). These variables were entered into an age and sex adjusted summary model. In this model, both age $(\beta=-0.30 ; \mathrm{P}=0.038)$ and the natural log of left ventricular ejection fraction $(\beta=0.50$; $\mathrm{P}=0.001$ ) were associated with MMSE score, after adjusting for potential confounders.

\section{Discussion}

Results of the present study indicate that cognitive impairment in patients with chronic heart failure is independently associated with both older age and decreased left ventricular systolic function. As previously reported, ${ }^{3-5} 10$ moderate to severe cognitive impairment in people with chronic heart failure is common, and mainly related to impairment in complex reasoning (calculation and visual-spatial intelligence). As previously reported for patients with chronic heart failure, ${ }^{21}$ depressive symptoms were common in the study population (table); this finding has been attributed to activation of the renin-angiotensin and sympathoadrenergic systems. ${ }^{21}$ However, depressive symptoms were not associated with cognitive performance. The prevalence of cognitive impairment in our patients with mild to moderate heart failure was similar to that found in younger subjects with end stage heart failure. ${ }^{45}$ This finding may be explained by the older age of participants in the present study, as age was associated with worsening cognitive performance in this study, as well as in previous studies on younger patients with heart failure. ${ }^{5}$ The pathophysiological determinants of cognitive impairment in patients with heart failure are uncertain. The highest prevalence of cognitive deficit in our patients was found on tests of complex reasoning; the same pattern of deficit has been found in previous studies on younger patients who were awaiting cardiac transplantation, and attributed to frontal and temporal lobe dysfunction. ${ }^{45}$ Nevertheless, no signs of damage to these areas have been so far seen on $\mathrm{CT}^{3}$ Despite the exclusion of subjects with abnormal Hachinski ischaemic score, the hypothesis of cerebral embolic vascular disease in our patients cannot be ruled out. However, the reported incidence of systemic embolism in subjects with heart failure is far too low to account for the frequent development of cognitive dysfunction in our patients. ${ }^{22}$ The finding of an apparent decline in cognitive function indices in subjects with low ejection fraction (figure) supports the role of reduced cerebral blood flow. ${ }^{3-10}$ Accordingly, the age related differences in cognitive performance of patients with heart failure may be ascribed to the impairment in autoregulatory mechanisms of cerebral circulation which has been reported in older subjects. ${ }^{23}$ Noteworthy, white matter lesions have been described on CT in older subjects, and associated with heart failure and systolic hypotension; dementia was a frequent finding in such patients. ${ }^{24}$ Also, subcortical alterations have been associated with chronic cerebral hypoperfusion in a neuropathological study. ${ }^{25}$ Indeed, none of our patients exhibited signs or symptoms of subcortical dysfunction, such as gait disorders, dysarthria, or urinary incontinence. ${ }^{24}{ }^{26}$ However, systematic neuroradiological evaluation was not performed, so that involvement of subcortical areas cannot be ruled out. Improvement in cognitive performance has been found after cardiac transplantation and pacemaker implantation, ${ }^{38}$ but other studies contradicted this finding. ${ }^{5}{ }^{10}$ Duration of reduction in cerebral blood flow may represent the explanation for this discrepancy, as both clinical and pathological studies have suggested that prolonged cerebral hypoperfusion may lead to degenerative brain abnormalities, including subcortical lesions. ${ }^{24}{ }^{25}$ In this setting, definite answers may only be derived from prospective studies, which can overcome the intrinsic limitations (such as selection or report bias) of cross sectional studies such as ours. Systematic neuropsychological testing of older patients with heart failure for early diagnosis of cognitive impairment might identify those who may most benefit from prompt echocardiographic evaluation and aggressive treatment of left ventricular dysfunction. Such a multidisciplinary approach to older patients with heart failure may play a key part in reducing the burden of so called "circulatory dementia" in advanced age, as the prevalence and incidence of heart failure are rapidly increasing, and substantial decreases in cognitive function have been associated in general populations with diagnosis of cardiovascular diseases. ${ }^{27}$ However, studies are still needed to assess the impact of treatments proved to prevent deterioration of left ventricular function (such as ACE inhibitors, digoxin, or $\beta$ blockers) on the incidence and rate of decline of cognitive ability in patients with heart failure.

1 Gillum RF. Heart failure in the United States 1970-85. Am Heart f 1987;113:1043-5.

2 Ghali JK, Cooper R, Ford E. Trends in hospitalization rates for heart failure in the United States, 1973-86. Arch Intern Med 1990;150:769-73.

3 Bornstein RA, Starling RC, Myerowitz P, Haas GJ. Neuropsychological function in patients with end-stage heart failure before and after cardiac transplantation. Acto Neurol Scand 1995;91:260-5.

4 Gorkin L, Norvell NK, Rosen RC, et al. Assessment of quality of life as observed from the baseline data of the studies of left ventricular dysfunction (SOLVD) trial quality of life of left ventricular dysfunction (SOLVD)
study. Am f Cardiol 1993;71:1069-73.

5 Scahll RR, Petrucci RJ, Brozena SC, Cavarocchi NC, Jessup M. Cognitive function in patients with symptomatic dilated cardiomyopathy before and after cardiac transplantation. $\mathcal{F}$ Am Coll Cardiol 1989;14:1666-72.

6 Rosemberg GA, Haaland RY. Cardiogenic dementia. Lancet 1981;ii: 1171 .

7 Editorial. Cardiogenic dementia. Lancet 1977;i:27-8.

8 Koide H, Kobayashi S, Kitani M, Tsunematsu T, Nakazawa Y. Improvement of cerebral blood flow and cognitive function following pacemaker implantation in patients with tion following pacemaker implantation
bradycardia. Gerontology 1994;40:79-85.

9 Barclay LL, Weiss EM, Mattis S, Bond O, Blass JP. Unrecognized cognitive impairment in cardiac rehabilitation patients. F Am Geriatr Soc 1988;36:22-8. 
10 Rockwood K, Dobbs AR, Rule BG, Howlett SE, Black WR The impact of pace-maker implantation on cognitive func-

11 McKhann G, Drachman D, Folstein M, et al. Clinical diagnosis of Alzheimer's disease: report of the NINCDSADRDA work group under the auspices of Department of Health and Human Services Task force on Alzheimer's disease. Neurology 1983;34:939-44.

12 Hachinski V, Lassen N, Marshall J. Multi-infarct dementia: a cause of mental deterioration in the elderly. Lancet 1974; ii: $207-10$.

13 Folstein MF, Folstein SE, McHugh P. Mini mental state: a practical method for grading the cognitive state of patients for the clinician. F Psychol Res 1975;12:189-98.

14 Caltagirone C, Gainotti G, Masullo C, Miceli G. Validity of some neuropsychological tests in the assessment of mental deterioration. Acta Psychiatr Scand 1979;60:50-6.

15 Raven JC. Progressive matrices (1947), Sets A, Ab, B: board and book forms. London: Lewis, 1949.

16 Radloff LS. The CES-D score: a self-report depression scale for research in the general population. Appl Psychol Meas for research in the

17 Davidson H, Feldman PH, Crawford S. Measuring depressive symptoms in the frail elderly. F Gerontol 1994;49:15964.

18 Katz S. Assessing self-maintenance: activities of daily living mobility, and instrumental activities of daily living. $7 \mathrm{Am}$ Geriatr Soc 1983;31:721-7.
19 Lawton MP, Brody EM. Assessment of older: selfmaintaining and instrumental activities of daily living. Germaintaining and instrum

20 Carlson KJ, Lee DC, Goroll AH, et al. An analysis of physicians' reasons for prescribing long-term digitalis therapy in out-patients. F Chron Dis 1985;38:733-9.

21 Zuccalà G, Cocchi A, Carbonin PU. The impact of depression on self-perceived health status. 7 Am Geriatr Soc 1995; 43:198-9.

22 Katz SD, Marantz PR, Biasucci L, et al. Low incidence of stroke in ambulatory patients with heart failure: a prospecstroke in ambulatory patients with heart
tive study. Am Heart $\mathscr{f}$ 1993;126:141-6.

23 Melamed E, Lavy S, Bentin S, Cooper G, Rinot Y. Reduction in regional cerebral blood flow during normal aging in man. Stroke 1980;11:31-5.

24 Tavornen-Schroder S, Roytta M, Raiha I, et al. Clinical features of leuko-araiosis. $\mathcal{F}$ Neurol Neurosurg Psychiatry 1996; 60:431-6.

25 Brun A, Englund E. A white matter disorder in dementia of the Alzheimer type: a pathoanatomical study. Ann Neurol 1986;19:253-62

26 Cummings JL. Vascular subcortical dementias: clinical aspects. Dementia 1994;5:177-80.

27 Breteler MMB, Claus JJ, Grobbee DE, Hofman A. Cardiovascular disease and distribution of cognitive function in elderly people: the Rotterdam study. BMf 1994;308: 1604-8. 